\title{
Many-body flatband localization
}

\author{
Carlo Danieli $\odot,{ }^{1}$ Alexei Andreanov, ${ }^{2,3}$ and Sergej Flach ${ }^{2,3,4}$ \\ ${ }^{1}$ Max Planck Institute for the Physics of Complex Systems, Dresden D-01187, Germany \\ ${ }^{2}$ Center for Theoretical Physics of Complex Systems, Institute for Basic Science (IBS), Daejeon 34126, Korea \\ ${ }^{3}$ Basic Science Program (IBS School), Korea University of Science and Technology (UST), Daejeon 34113, Korea \\ ${ }^{4}$ New Zealand Institute for Advanced Study, Massey University, Auckland, Private Bag 102904, 0632 Auckland, New Zealand
}

(Received 17 April 2020; accepted 29 June 2020; published 20 July 2020)

\begin{abstract}
We generate translationally invariant systems exhibiting many-body localization from all-bands-flat singleparticle lattice Hamiltonians dressed with suitable short-range many-body interactions. This phenomenon, dubbed many-body flatband localization, is based on symmetries of both single-particle and interaction terms in the Hamiltonian, and it holds for any interaction strength. We propose a generator of corresponding Hamiltonians which covers both interacting bosons and fermions for arbitrary lattice dimensions, and we provide explicit examples of such models in one and two lattice dimensions. We also explicitly construct an extensive set of local integrals of motion for this set of models. Our results can be further generalized to long-range interactions as well as to systems lacking translational invariance.
\end{abstract}

DOI: 10.1103/PhysRevB.102.041116

Introduction. Understanding the lack of thermalization in quantum interacting systems has been an active topic since Anderson predicted in 1958 the absence of transport in singleparticle lattices due to spatial disorder [1]. This localization phenomenon has been extensively studied theoretically and experimentally [2], with the impact of interaction between localized particles as one of the main open questions. Weak interactions were predicted to preserve the absence of transport of interacting particles $[3,4]$ about 50 years after Anderson's original work, leading to the phenomenon of many-body localization (MBL). The study of MBL systems and their properties is nowadays a very active topic of research with several open issues and active fronts (for a survey of the state of the art, see $[5,6])$.

MBL was initially predicted for interacting disordered systems emerging as an interplay of disorder and weak interactions. However, it was later realized that the presence of disorder is not essential, launching the search for disorder-free MBL systems. Several possible scenarios emerged as a result: from nonergodic behavior in networks of Josephson junctions [7] to one-dimensional (1D) fermionic lattices involving different species of particles [8] or the presence of DC field [9], local constraints due to gauge invariance [10], the presence of a large number of conserved quantities [11,12], quasiperiodic long-range interactions [13], among others. Some proposals also explored the connection to glasses, predicting manybody localization in glassy systems [14-17], kinetically constrained models [18], and geometrically frustrated models

Published by the American Physical Society under the terms of the Creative Commons Attribution 4.0 International license. Further distribution of this work must maintain attribution to the author(s) and the published article's title, journal citation, and DOI. Open access publication funded by the Max Planck Society.
[19]. However, the validity of some of the proposals was later doubted, as it was shown that several disorder-free MBL systems rely on vastly different energy scales and finite-size constraints [20]. In other cases instead (e.g., [7]), disorder-free MBL requires high temperatures or specific strong interaction regimes; likewise the original MBL requests weak interaction regimes.

In this Rapid Communication, we propose a generator of disorder-free MBL systems which is free of the abovementioned requirements (specific interaction or temperature regimes, finite-size constraints, type of many-body statistics, among others) and applies for arbitrary spatial dimensions. This generator relies on geometrical frustration of the translationally invariant single-particle Hamiltonians which yields no single-particle dispersion, i.e., all Bloch bands are dispersionless (or flat), and suitably chosen many-body interactions. The resulting models exhibit nonergodic behavior with lack of transport of particles for any interaction strength, and this phenomenon is dubbed many-body flatband localization (MBFBL). The study of networks with one or several flatbands is an active topic of research on its own. They were first discussed in the context of ground-state ferromagnetism [21], but were later identified in various other systems [22,23] and they have been experimentally realized in several setups, using, e.g., ultracold atoms [24] and photonic lattices [25-27]. An important property of flatband systems is the presence of compact localized states_-eigenstates with strictly finite support. These were used to systematically construct flatbands [28-30] along with other methods [31-35]. Their fine-tuned character makes flatband systems an ideal platform to study diverse localization phenomena in the presence of on-site disorder [36-39], DC fields [40], and nonlinearities [41,42], among many others.

We introduce MBFBL networks formed by single-particle all-bands-flat lattice Hamiltonians dressed with suitable shortrange many-body interactions, and provide explicit examples 
in one and two spatial dimensions. We also discuss distinct interaction terms (including long-range interactions) in order to cover different types of particle statistics. We construct an extensive set of local integrals of motion present in MBFBL networks, and explicitly derive these integrals for some of the examples presented. We extend our generator scheme by removing the assumption of translation invariance of the lattice.

Setup. We consider a translationally invariant many-body Hamiltonian $\hat{\mathcal{H}}$ on a lattice

$$
\hat{\mathcal{H}}=\hat{\mathcal{H}}_{\mathrm{sp}}+\hat{\mathcal{H}}_{\text {int }}, \quad \hat{\mathcal{H}}_{\mathrm{sp}}=\sum_{k} \hat{f}_{k}, \quad \hat{\mathcal{H}}_{\text {int }}=\sum_{\kappa} \hat{g}_{\kappa}
$$

with both single-particle part $\hat{\mathcal{H}}_{\mathrm{sp}}$ and interaction $\hat{\mathcal{H}}_{\text {int }}$ written as sums of local operators $\hat{f}_{k}$ and $\hat{g}_{\kappa}$. The integers $k$ and $\kappa$ label unit cells of the lattice in a direct space for two different unit cell choices $A$ and $B$. We assume that the sites from one unit cell of, e.g., choice $A$ belong to different unit cells of choice $B$. Regardless of the choice, each unit cell contains $v$ lattice sites or single-particle levels. The operators are expressed through creation and annihilation operators $\hat{c}_{k, a}^{\dagger}, \hat{c}_{k, a}$ which create or annihilate a single particle on a given lattice site $k, a$ with $1 \leqslant$ $a \leqslant v$. Then the local operators read

$$
\hat{f_{k}}=\sum_{a, b=1}^{v} t_{a b} \hat{c}_{k, a}^{\dagger} \hat{c}_{k, b}+\text { H.c. }
$$

We assume the interaction Hamiltonian $\hat{\mathcal{H}}_{\text {int }}$ to be two-body, so that the local operators are

$$
\hat{g}_{\kappa}=\sum_{\alpha, \beta, \gamma, \delta=1}^{\nu} J_{\alpha \beta \gamma \delta} \hat{c}_{\kappa, \alpha}^{\dagger} \hat{c}_{\kappa, \beta}^{\dagger} \hat{c}_{\kappa, \gamma} \hat{c}_{\kappa, \delta}+\text { H.c. }
$$

By the above definitions both single-particle and interaction Hamiltonians are semidetangled (SD) as $\left[\hat{f}_{k}, \hat{f}_{k^{\prime}}\right]=$ $\left[\hat{g}_{\kappa}, \hat{g}_{\kappa^{\prime}}\right]=0$ for any $k, k^{\prime}, \kappa, \kappa^{\prime}$. The spectrum of the singleparticle eigenvalue problem with $\hat{\mathcal{H}}_{\text {sp }}$ yields $v$ flatbands with each being an eigenenergy of any of the local operators $f_{k}$. It follows that $\hat{\mathcal{H}}_{\mathrm{sp}}$ enforces full localization and absence of transport. The same is true for $\hat{\mathcal{H}}_{\text {int }}$. However, because of the different unit cell choices $A, B$, in general it follows that $\left[\hat{f}_{k}, \hat{g}_{\kappa}\right] \neq 0$ for any given $k$ and at least a pair of different values of $\kappa$ (and vice versa). Consequently, the combination of both $\hat{\mathcal{H}}_{\mathrm{sp}}$ and $\hat{\mathcal{H}}_{\text {int }}$ into $\mathcal{H}$ in general yields transporting many-body eigenstates [43-46].

If $t_{a b}=t_{a a} \delta_{a, b}$ (with the Kronecker symbol $\delta_{a, b}$ ), the $\hat{\mathcal{H}}_{\mathrm{sp}}$ is coined fully detangled (FD) [31] since it depends on the particle number operators $\hat{n}=\hat{c}^{\dagger} \hat{c}$ only, and does not move any particles from any lattice site to any other one. Together with $\hat{\mathcal{H}}_{\text {int }}$ being SD, the full Hamiltonian $\mathcal{H}$ preserves full localization of particles, which is an example of MBFBL. Likewise, if we assume that $J_{\alpha \beta \gamma \delta}=J_{\alpha \beta \alpha \beta} \delta_{\alpha, \gamma} \delta_{\beta, \delta}$ it follows that $\hat{\mathcal{H}}_{\text {int }}$ is FD and does not move any particles from site to site. Together with $\hat{\mathcal{H}}_{\text {sp }}$ being SD, we again arrive at the result that the full Hamiltonian $\mathcal{H}$ lacks transporting eigenstates and is MBFBL. The relation between the character of the Hamiltonians $\hat{\mathcal{H}}_{\text {sp }}, \hat{\mathcal{H}}_{\text {int }}$ and the presence of MBFBL is summarized in Table I. We refer to all the other types of Hamiltonians as nondetangled.
TABLE I. Existence of MBFBL for different types of singleparticle Hamiltonian $\hat{\mathcal{H}}_{\text {sp }}$ and interaction Hamiltonian $\hat{\mathcal{H}}_{\text {int }}$ as discussed in the main text.

\begin{tabular}{lcc}
\hline \hline$\hat{\mathcal{H}}_{\text {sp }} / \hat{\mathcal{H}}_{\text {int }}$ & SD & FD \\
\hline SD & & MBFBL \\
FD & MBFBL & MBFBL \\
\hline \hline
\end{tabular}

We generate MBFBL Hamiltonians by choosing any of the three available combinations from Table I. We then perform a unitary transformation (rotation) on each unit cell in either of the two unit cell choices $A, B$. This results in general in some complicated Hamiltonian with $\hat{\mathcal{H}}_{\mathrm{sp}}$ being nondetangled and $\hat{\mathcal{H}}_{\text {int }}$ being fully or semidetangled, or vice versa [47] depending on which unit cell type the transformation was applied to. Furthermore these transformations can be chosen unit cell dependent resulting in nontranslationally invariant Hamiltonians.

Conventional disordered MBL systems are known to possess an extensive set of local integrals of motion [5,48,49], though explicit derivations are complicated. These integrals are used to explain relevant properties of these systems. Local integrals of motion can be explicitly derived for MBFBL networks. With our proposed scheme and considering a semidetangled single-particle Hamiltonian $\hat{\mathcal{H}}_{\text {sp }}$ in $\mathcal{H}(1)$, it follows that the expectation values of the operators $\hat{I}_{k}=\sum_{a=1}^{v} \hat{n}_{k, a}$ measure the number of particles in each local unit $\hat{f}_{k}$ of $\hat{\mathcal{H}}_{\text {sp. }}$. These numbers are conserved in the presence of a fully detangled interaction $\hat{\mathcal{H}}_{\text {int }}$ (since $\hat{\mathcal{H}}_{\text {int }}$ does not move particles from one to another site). It follows that each $\hat{I}_{k}$ commutes with the full Hamiltonian.

The unitary transformations used to recast $\hat{\mathcal{H}}_{\mathrm{sp}}$ as nondetangled yield $N$ local integrals of motion $\hat{I}_{k}$ expressed in the new basis for the generated lattice. The very same follows if a pair of FD single-particle $\hat{\mathcal{H}}_{\text {sp }}$ and a SD interaction $\hat{\mathcal{H}}_{\text {int }}$ is picked from Table I. In this case, the operators $\hat{I}_{\kappa}=\sum_{\alpha=1}^{\nu} \hat{n}_{\kappa, \alpha}$ defined in each local unit $\hat{g}_{\kappa}$ of $\hat{\mathcal{H}}_{\text {int }}$ as well lead to $N$ local integrals of motion of the generated lattice after the unitary transformations have been applied. In the third case of Table I with both $\hat{\mathcal{H}}_{\mathrm{sp}}, \hat{\mathcal{H}}_{\text {int }}$ fully detangled, the extensive set of local integrals of motion contains $v \times N$ elements, since each particle number operator $\hat{n}_{k, a}$ commutes with the full Hamiltonian $\mathcal{H}$. In that third case neither particles nor heat (energy) are transported across the system.

Most of the generated MBFBL models, while being appealing from a mathematical point of view, could be hard to implement in experiments due to the complicated structure of the interaction $\hat{\mathcal{H}}_{\text {int }}$ spanning several unit cells. Experimental feasibility instead favors fully detangled $\hat{\mathcal{H}}_{\text {int }}$, which result, e.g., from Coulomb interactions between density operators in real space [50]. Therefore we refine our scheme by choosing semidetangled single-particle $\hat{\mathcal{H}}_{\text {sp }}$ and fully detangled interaction $\hat{\mathcal{H}}_{\text {int }}$, and recast $\hat{\mathcal{H}}_{\text {sp }}$ to a nondetangled Hamiltonian via unitary transformations that keep $\hat{\mathcal{H}}_{\text {int }}$ fully detangled. This algorithm works for any number of bands $v$ of $\hat{\mathcal{H}}_{\mathrm{sp}}$, in any dimension, and any type of many-body statistics. 
Results. We will now discuss concrete examples in one and two spatial dimensions. We consider the semidetangled Hamiltonian $\hat{\mathcal{H}}_{\mathrm{sp}}$ and conveniently restate it in the unit cell representation $B$ of $\hat{\mathcal{H}}_{\text {int }}$. We then apply the subsequent unitary transformations. This change of unit cell introduces hopping terms between neighboring unit cells in each local Hamiltonian $\hat{f}_{\kappa}$. Without loss of generality, we assume nonzero hoppings between nearest-neighboring unit cells only, and we adopt the conventions used in Refs. [28,29] for flatband network generators. Then a possible $D=1$ Hamiltonian $\hat{\mathcal{H}}_{\mathrm{sp}}$ reads

$$
\hat{\mathcal{H}}_{\mathrm{sp}}=\sum_{\kappa} \hat{f}_{\kappa}=\sum_{\kappa}\left[\frac{1}{2} \hat{C}_{\kappa}^{\dagger T} H_{0} \hat{C}_{\kappa}+\hat{C}_{\kappa}^{\dagger T} H_{1} \hat{C}_{\kappa+1}+\text { H.c. }\right] \text {, }
$$

where we grouped the annihilation (creation) operators $\hat{c}_{\kappa, a}$ $\left(\hat{c}_{\kappa, a}^{\dagger}\right)$ in $v$-dimensional vectors $\hat{C}_{\kappa}\left(\hat{C}_{\kappa}^{\dagger}\right)$. The matrices $H_{0}, H_{1}$ describe intra- and intercell hopping, respectively, and are chosen so as to enforce the SD condition $\left[\hat{f}_{\kappa}, \hat{f}_{\kappa^{\prime}}\right]=0$ for all $\kappa, \kappa^{\prime}$. We remark that this $\hat{\mathcal{H}}_{\text {sp }}$ is only one of the infinitely many realizations of a SD single-particle Hamiltonian.

The fully detangled two-body interaction Hamiltonian $\hat{\mathcal{H}}_{\text {int }}$ introduced above [(1), (3)] is taken with the coefficients $J_{\alpha \beta \gamma \delta}=J_{\alpha \beta \alpha \beta} \delta_{\alpha, \gamma} \delta_{\beta, \delta}$ for each local component $\hat{g}_{\kappa}: J_{\alpha \beta \alpha \beta}=1$ for $\alpha=\beta$ and $J_{\alpha \beta \alpha \beta}=2$ for $\alpha \neq \beta$. Then $\hat{\mathcal{H}}_{\text {int }}$ is preserved as fully detangled with the same coefficients $J_{\alpha \beta \gamma \delta}$ by any $2 \times 2$ unitary transformation

$$
U_{a b}:\left\{\begin{array}{l}
\hat{c}_{\kappa, a}=z \hat{d}_{\kappa, a}+w \hat{d}_{\kappa, b} \\
\hat{c}_{\kappa, b}=-w^{*} \hat{d}_{\kappa, a}+z^{*} \hat{d}_{\kappa, b}
\end{array}\right.
$$

parametrized by two complex numbers $z, w$ such that $|z|^{2}+$ $|w|^{2}=1$ and any pair of sites $\hat{c}_{\kappa, a}, \hat{c}_{\kappa, b}$.

The resulting Hamiltonian $\hat{\mathcal{H}}_{\text {int }}$ for $v=2$ bands describes a two-body interaction among the sites $\hat{a}_{\kappa}=\hat{c}_{\kappa, a}, \hat{b}_{\kappa}=\hat{c}_{\kappa, b}$,

$$
\begin{aligned}
\hat{\mathcal{H}}_{\text {int }} & =\sum_{\kappa}\left[\hat{a}_{\kappa}^{\dagger} \hat{a}_{\kappa}^{\dagger} \hat{a}_{\kappa} \hat{a}_{\kappa}+\hat{b}_{\kappa}^{\dagger} \hat{b}_{\kappa}^{\dagger} \hat{b}_{\kappa} \hat{b}_{\kappa}+2 \hat{a}_{\kappa}^{\dagger} \hat{a}_{\kappa} \hat{b}_{\kappa}^{\dagger} \hat{b}_{\kappa}\right] \\
& =\sum_{\kappa}\left[\hat{n}_{a, \kappa}+\hat{n}_{b, \kappa}-1\right]\left[\hat{n}_{a, \kappa}+\hat{n}_{b, \kappa}\right]
\end{aligned}
$$

with $\hat{n}_{a, \kappa}=\hat{a}_{\kappa}^{\dagger} \hat{a}_{\kappa}$ and $\hat{n}_{b, \kappa}=\hat{b}_{\kappa}^{\dagger} \hat{b}_{\kappa}$. We refer to such interaction as an extended Hubbard interaction, which applies to both bosons and fermions with spin.

$1 D$ networks. We now present two concrete examples. We first start with the simplest MBFBL network with $v=2$ bands. It is based on the Hamiltonian $\hat{\mathcal{H}}_{\mathrm{sp}}$ in Eq. (4) with

$$
H_{0}=\left(\begin{array}{ll}
1 & 0 \\
0 & 0
\end{array}\right), \quad H_{1}=\left(\begin{array}{cc}
0 & t \\
0 & 0
\end{array}\right),
$$

and a free complex parameter $t$. It is straightforward to check that this Hamiltonian is semidetangled and has all bands flat. Next we pick the extended Hubbard interaction $\hat{\mathcal{H}}_{\text {int }}(6)$. The structure of $\hat{\mathcal{H}}_{\text {sp }}$ and $\hat{\mathcal{H}}_{\text {int }}$ is shown in Fig. 1(a) with solid lines and red-shaded rods, respectively. The rotation $U_{a b}(5)$ recasts $H_{0}, H_{1}(7)$ as

$$
H_{0}=\left(\begin{array}{cc}
|z|^{2} & z^{*} w \\
z w^{*} & |w|^{2}
\end{array}\right), \quad H_{1}=t\left(\begin{array}{cc}
-z^{*} w^{*} & \left(z^{*}\right)^{2} \\
-\left(w^{*}\right)^{2} & z^{*} w^{*}
\end{array}\right),
$$

and makes Hamiltonian $\hat{\mathcal{H}}_{\text {sp }}$ nondetangled while $\hat{\mathcal{H}}_{\text {int }}$ remains fully detangled. The resulting MBFBL network is shown in

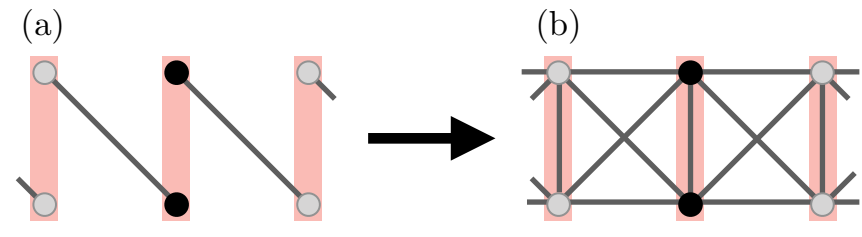

FIG. 1. One-dimensional two-band MBFBL network with $\hat{\mathcal{H}}_{\mathrm{sp}}$ SD (a) and with the cross-stitch lattice profile (b). The black circles indicate the unit cell choice, the solid lines correspond to sites connected by $\hat{\mathcal{H}}_{\mathrm{sp}}$ before (a) and after (b) the rotation, and the redshaded rods indicate the sites connected by the extended Hubbard terms (6) of $\hat{\mathcal{H}}_{\text {int }}$.

Fig. 1(b). The local integrals of motion read (after the rotation)

$$
\begin{aligned}
\hat{I}_{\kappa}= & |z|^{2}\left(\hat{n}_{a, \kappa-1}+\hat{n}_{b, \kappa}\right)+|w|^{2}\left(\hat{n}_{a, \kappa}+\hat{n}_{b, \kappa-1}\right) \\
& +z^{*} w\left(\hat{a}_{\kappa-1}^{\dagger} \hat{b}_{\kappa-1}-\hat{a}_{\kappa}^{\dagger} \hat{b}_{\kappa}\right)+\text { H.c. }
\end{aligned}
$$

For three bands $v=3$ with operators $a_{\kappa}, b_{\kappa}, c_{\kappa}$ corresponding to the three sites of the unit cell, the semidetangled Hamiltonian $\hat{\mathcal{H}}_{\mathrm{sp}}(6)$ has the following hopping matrices:

$$
H_{0}=\left(\begin{array}{ccc}
1 & 0 & 0 \\
0 & 0 & t_{1} \\
0 & t_{1}^{*} & \mu
\end{array}\right), \quad H_{1}=\left(\begin{array}{ccc}
0 & 0 & t_{2} \\
0 & 0 & 0 \\
0 & 0 & 0
\end{array}\right),
$$

with two free complex $\left(t_{1}, t_{2}\right)$ and one free real $(\mu)$ parameters. This network is shown in Fig. 2(a) with gray solid lines. The interaction $\hat{\mathcal{H}}_{\text {int }}$ consists of the extended Hubbard interaction (6) between the top and the bottom sites $\left(a_{\kappa}, b_{\kappa}\right)$ of each plaquette [red-shaded rods in Fig. 2(a)] and an additional optional on-site Hubbard interaction for the central site $c_{\kappa}$. Then the rotation $U_{a b}(5)$ is applied to the pair $\left(a_{\kappa}, b_{\kappa}\right)$ only while leaving the sites $c_{\kappa}$ untouched. This recasts $H_{0}, H_{1}$ (10) into

$$
H_{0}=\left(\begin{array}{ccc}
|z|^{2} & z^{*} w & -t_{1} w \\
z w^{*} & |w|^{2} & t_{1} z \\
-t_{1}^{*} w^{*} & t_{1}^{*} z^{*} & \mu
\end{array}\right), \quad H_{1}=t_{2}\left(\begin{array}{ccc}
0 & 0 & z^{*} \\
0 & 0 & w^{*} \\
0 & 0 & 0
\end{array}\right)
$$

defining a nondetangled Hamiltonian $\hat{\mathcal{H}}_{\mathrm{sp}}$ while the interaction $\hat{\mathcal{H}}_{\text {int }}$ remains fully detangled. The resulting diamondshaped MBFBL network is shown in Fig. 2(b). That diamondshape profile has been realized in diverse experimental setups for flatband and compact localized state studies [51-55]. Experimentally, the selective extended Hubbard interaction involving only the top and bottom sites $\hat{a}_{\kappa}, \hat{b}_{\kappa}$ of the diamond

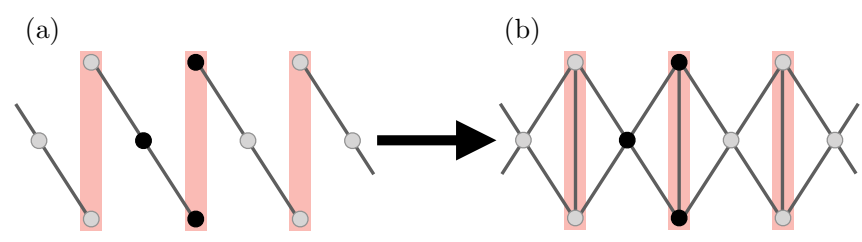

FIG. 2. One-dimensional three-band MBFBL network with $\hat{\mathcal{H}}_{\mathrm{sp}}$ $\mathrm{SD}$ (a) and with the diamond-shaped lattice profile (b). The black circles indicate the unit cell choice, the solid lines correspond to $\hat{\mathcal{H}}_{\mathrm{sp}}$ before (a) and after (b) the rotations, and the red-shaded rods indicate the extended Hubbard terms (6) of $\hat{\mathcal{H}}_{\text {int }}$. 
(a)

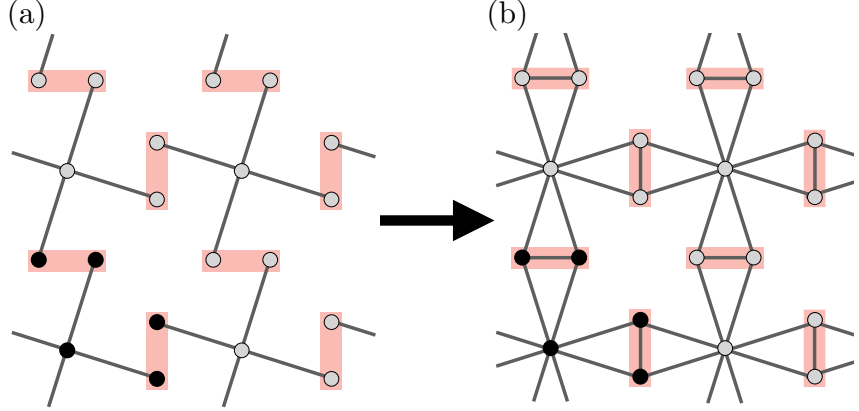

FIG. 3. Two-dimensional five-band MBFBL network with $\hat{\mathcal{H}}_{\mathrm{sp}}$ SD (a) and with the decorated Lieb lattice profile (b). The black circles indicate the unit cell choice, the solid lines correspond to $\hat{\mathcal{H}}_{\mathrm{sp}}$ before (a) and after (b) the rotations, and the red-shaded rods indicate the extended Hubbard terms (6) of $\hat{\mathcal{H}}_{\text {int }}$.

plaquette might be achieved by reducing the distance between these sites as compared to the distance to the middle site $\hat{c}_{\kappa}$. The parameter $t_{1}$ could be used to adjust the hoppings. The local integrals of motion $\hat{I}_{\kappa}$ for this model are given by Eq. (9) plus the additional particle number operator $\hat{n}_{c, \kappa}$ for the central site $\hat{c}_{\kappa}$ of the lattice, since it is unaffected by the rotation.

$2 D$ networks. The construction of higher-dimensional MBFBL networks follows a procedure similar to that of 1D systems. In the simplest setting, the single-particle Hamiltonian $\hat{\mathcal{H}}_{\text {sp }}$ can be taken as a straightforward extension of Eq. (4), where matrices $H_{1}$ are replaced with matrices $H_{0}$ and $H_{1}^{(1)}, \ldots, H_{1}^{(D)}$ describing the intercell hopping along different spatial directions. The matrices are chosen to ensure that $\hat{\mathcal{H}}_{\text {sp }}$ is semidetangled. Now taking a suitable FD interaction $\hat{\mathcal{H}}_{\text {int }}$, Eq. (6) or its generalizations, and picking a unitary transformation that leaves this $\hat{\mathcal{H}}_{\text {int }}$ fully detangled, the full Hamiltonian $\mathcal{H}$ exhibits MBFBL [56].

A notable two-dimensional lattice exhibiting MBFBL obtained is the decorated Lieb lattice [57]. This is a five-band $v=5$ network, whose SD Hamiltonian $\hat{\mathcal{H}}_{\mathrm{sp}}$ is shown in Fig. 3(a), and defined with matrices $H_{0}, H_{1}^{(1)}, H_{1}^{(2)}$. In each unit cell, we use the extended Hubbard Hamiltonians $\hat{\mathcal{H}}_{\text {int }}(6)$ for the two site pairs indicated by red-shaded rods in Fig. 3(a), and an on-site Hubbard interaction for the central site. The two rotations $U_{a b}(5)$ applied to the highlighted pairs (leaving the central site untouched) yield a nondetangled $\hat{\mathcal{H}}_{\text {sp }}$ shown in Fig. 3(b), and the resulting full Hamiltonian $\mathcal{H}$ is MBFBL. The local integrals of motion for the decorated Lieb lattice can be easily derived and have similar but more involved expressions to those of the previous models (9).

Perspectives. The proposed scheme relies on the two-body Hamiltonian $\hat{\mathcal{H}}_{\text {int }}$ with on-site terms in the interaction, restricting the interacting particles to bosons or spinful fermions. However, the same construction can be implemented for spinless fermions by, e.g., choosing local operators $\hat{g}_{\kappa}^{\sigma}=$ $\sum_{\alpha, \beta, \gamma, \delta=1}^{\nu} J_{\alpha \beta \gamma \delta}^{\sigma} \hat{c}_{\kappa, \alpha}^{\dagger} \hat{c}_{\kappa+\sigma, \beta}^{\dagger} \hat{c}_{\kappa, \gamma} \hat{c}_{\kappa+\sigma, \delta}+$ H.c. with exclusively intersite interaction terms between unit cell $\kappa$ and unit cell $\kappa+$ $\sigma$. In particular, $\hat{\mathcal{H}}_{\text {int }}$ is fully detangled for $J_{\alpha \beta \gamma \delta}^{\sigma}=J \delta_{\alpha, \gamma} \delta_{\beta, \delta}$ and it is preserved as such by the same transformation (5).
This yields a generator of $D$-dimensional $v$-band MBFBL lattices for spinless fermions, with the recent work of Kuno et al. [58] being a particular $D=1, v=2$ band example. The construction can be further extended to long-range all-to-all interaction Hamiltonians $\hat{\mathcal{H}}_{\text {int }}$ by setting $\hat{g}_{\kappa}=\sum_{\sigma} v_{\sigma} \hat{g}_{\kappa}^{\sigma}$ and even infinite-range interactions $\hat{\mathcal{H}}_{\text {int }}=J / N \sum_{\kappa \neq \kappa^{\prime}, a} \hat{n}_{\kappa, a} \hat{n}_{\kappa^{\prime}, a}$. The latter example is valid because the interaction is a function of the total density $\hat{\rho}=\sum_{\kappa, a} \hat{n}_{\kappa, a}$ only and is therefore invariant under the transformation (5).

We note that it is possible to extend the generator by abandoning the translational invariance of the Hamiltonian $\mathcal{H}$. We can choose the hopping parameters $t_{a b}$ and the interaction matrix elements $J_{\alpha \beta \gamma \delta}$ in the starting Hamiltonians $\hat{\mathcal{H}}_{\mathrm{sp}}$ and $\hat{\mathcal{H}}_{\text {int }}$, respectively, to be unit cell dependent. To stick with the proposed scheme where $\hat{\mathcal{H}}_{\text {int }}$ is fully detangled and is preserved by unitary transformations (5), the unit-cell-dependent terms are restrained to the semidetangled $\hat{\mathcal{H}}_{\text {sp }}$ only (e.g., onsite or hopping disorder). The unitary transformations (5) used to recast $\hat{\mathcal{H}}_{\text {sp }}$ as nondetangled induce correlations between the on-site energies of the pairs of sites involved. In the models presented [Figs. 1(b), 2(b), and 3(b)] these correlations are between the sites within the same red-shaded area. These correlations depend on the parameters $z, w$ defining $U_{a, b}$ in Eq. (5). These parameters may also be chosen to vary upon changing $\kappa$ if the unitary transformations considered differ from unit cell to unit cell. Let us additionally observe that the breaking of translation invariance does not destroy the existence of the extensive set of local integrals of motion; they are given by the same operators as in the translationally invariant case.

Conclusions. We have introduced a generator of manybody localized disorder-free Hamiltonians by applying unitary transformations to suitably detangled Hamiltonians-a feature that assumes all-band-flat single-particle Hamiltonians. This phenomenon, coined many-body flatband localization, implies strict localization of particles (interaction or single-particle Hamiltonian is semidetangled) and in addition heat (both are fully detangled) irrespective of dimensionality or interaction strength, and it does not require vastly different energy scales similar to some models supposed to exhibit disorder-free MBL. Our work substantially extends previous studies of localization phenomena of interacting quantum many-body platforms with all-band-flat lattice single-particle Hamiltonians [44,46,58-64]. In particular, we propose a flexible and general set of many-body localized systems which may be experimentally feasible. A novel and unique feature of these systems is the existence of unitary mappings that recast them into a detangled form. This very property can be employed to study the impact of additional perturbations of the proposed networks which lift MBFBL and modify the proposed local integrals of motion in a systematic and analytical form. Hence, these systems offer innovative and powerful tools to potentially perform systematic analytical studies of conventional properties of MBL networks which typically relay on heavy numerical studies.

Acknowledgments. This work was supported by the Institute for Basic Science (Project No. IBS-R024-D1). S.F. acknowledges support by the New Zealand Institute for Advanced Study where part of this work was completed. 
[1] P. W. Anderson, Absence of Diffusion in Certain Random Lattices, Phys. Rev. 109, 1492 (1958).

[2] B. Kramer and A. MacKinnon, Localization: Theory and experiment, Rep. Prog. Phys. 56, 1469 (1993).

[3] D. M. Basko, I. L. Aleiner, and B. L. Altshuler, Metalinsulator transition in a weakly interacting many-electron system with localized single-particle states, Ann. Phys. 321, 1126 (2006).

[4] I. L. Aleiner, B. L. Altshuler, and G. V. Shlyapnikov, A finite-temperature phase transition for disordered weakly interacting bosons in one dimension, Nat. Phys. 6, 900 (2010).

[5] D. A. Abanin and Z. Papić, Recent progress in many-body localization, Ann. Phys. 529, 1700169 (2017).

[6] D. A. Abanin, E. Altman, I. Bloch, and M. Serbyn, Colloquium: Many-body localization, thermalization, and entanglement, Rev. Mod. Phys. 91, 021001 (2019).

[7] M. Pino, L. B. Ioffe, and B. L. Altshuler, Nonergodic metallic and insulating phases of Josephson junction chains, Proc. Natl. Acad. Sci. USA 113, 536 (2016).

[8] M. Schiulaz, A. Silva, and M. Müller, Dynamics in many-body localized quantum systems without disorder, Phys. Rev. B 91, 184202 (2015).

[9] M. Schulz, C. A. Hooley, R. Moessner, and F. Pollmann, Stark Many-Body Localization, Phys. Rev. Lett. 122, 040606 (2019).

[10] P. Karpov, R. Verdel, Y. P. Huang, M. Schmitt, and M. Heyl, Disorder-free localization in an interacting two-dimensional lattice gauge theory, arXiv:2003.04901.

[11] A. Smith, J. Knolle, D. L. Kovrizhin, and R. Moessner, Disorder-Free Localization, Phys. Rev. Lett. 118, 266601 (2017).

[12] A. Smith, J. Knolle, R. Moessner, and D. L. Kovrizhin, Dynamical localization in $\mathbb{Z}_{2}$ lattice gauge theories, Phys. Rev. B 97, 245137 (2018).

[13] R. Mondaini and Z. Cai, Many-body self-localization in a translation-invariant Hamiltonian, Phys. Rev. B 96, 035153 (2017).

[14] C. R. Laumann, A. Pal, and A. Scardicchio, Many-Body Mobility Edge in a Mean-Field Quantum Spin Glass, Phys. Rev. Lett. 113, 200405 (2014).

[15] C. L. Baldwin, C. R. Laumann, A. Pal, and A. Scardicchio, The many-body localized phase of the quantum random energy model, Phys. Rev. B 93, 024202 (2016).

[16] C. L. Baldwin, C. R. Laumann, A. Pal, and A. Scardicchio, Clustering of Nonergodic Eigenstates in Quantum Spin Glasses, Phys. Rev. Lett. 118, 127201 (2017).

[17] G. Mossi, T. Parolini, S. Pilati, and A. Scardicchio, On the quantum spin glass transition on the Bethe lattice, J Stat. Mech. (2017) 013102.

[18] M. van Horssen, E. Levi, and J. P. Garrahan, Dynamics of many-body localization in a translation-invariant quantum glass model, Phys. Rev. B 92, 100305(R) (2015).

[19] Y. Zhao and A. Andreanov (unpublished).

[20] Z. Papić, E. M. Stoudenmire, and D. A. Abanin, Many-body localization in disorder-free systems: The importance of finitesize constraints, Ann. Phys. 362, 714 (2015).

[21] A. Mielke and H. Tasaki, Ferromagnetism in the Hubbard model, Commun. Math. Phys. 158, 341 (1993).
[22] D. Leykam, A. Andreanov, and S. Flach, Artificial flat band systems: From lattice models to experiments, Adv. Phys.: X 3, 1473052 (2018).

[23] D. Leykam and S. Flach, Perspective: Photonic flatbands, APL Photonics 3, 070901 (2018).

[24] S. Taie, H. Ozawa, T. Ichinose, T. Nishio, S. Nakajima, and Y. Takahashi, Coherent driving and freezing of bosonic matter wave in an optical Lieb lattice, Sci. Adv. 1, e1500854 (2015).

[25] S. Mukherjee, A. Spracklen, D. Choudhury, N. Goldman, P. Öhberg, E. Andersson, and R. R. Thomson, Observation of a Localized Flat-Band State in a Photonic Lieb Lattice, Phys. Rev. Lett. 114, 245504 (2015).

[26] R. A. Vicencio, C. Cantillano, L. Morales-Inostroza, B. Real, C. Mejía-Cortés, S. Weimann, A. Szameit, and M. I. Molina, Observation of Localized States in Lieb Photonic Lattices, Phys. Rev. Lett. 114, 245503 (2015).

[27] S. Weimann, L. Morales-Inostroza, B. Real, C. Cantillano, A. Szameit, and R. A. Vicencio, Transport in sawtooth photonic lattices, Opt. Lett. 41, 2414 (2016).

[28] W. Maimaiti, A. Andreanov, H. C. Park, O. Gendelman, and S. Flach, Compact localized states and flat-band generators in one dimension, Phys. Rev. B 95, 115135 (2017).

[29] W. Maimaiti, S. Flach, and A. Andreanov, Universal $d=1$ flat band generator from compact localized states, Phys. Rev. B 99, 125129 (2019).

[30] W. M. Maimaiti, A. Andreanov, and S. Flach, Flatband generator in two dimension (unpublished).

[31] S. Flach, D. Leykam, J. D. Bodyfelt, P. Matthies, and A. S. Desyatnikov, Detangling flat bands into Fano lattices, Europhys. Lett. 105, 30001 (2014).

[32] R. G. Dias and J. D. Gouveia, Origami rules for the construction of localized eigenstates of the Hubbard model in decorated lattices, Sci. Rep. 5, 16852 (2015).

[33] A. Ramachandran, A. Andreanov, and S. Flach, Chiral flat bands: Existence, engineering, and stability, Phys. Rev. B 96, 161104(R) (2017).

[34] M. Röntgen, C. V. Morfonios, and P. Schmelcher, Compact localized states and flat bands from local symmetry partitioning, Phys. Rev. B 97, 035161 (2018).

[35] L. A. Toikka and A. Andreanov, Necessary and sufficient conditions for flat bands in $M$-dimensional $N$-band lattices with complex-valued nearest-neighbour hopping, J. Phys. A: Math. Theor. 52, 02 LT04 (2018).

[36] D. Leykam, S. Flach, O. Bahat-Treidel, and A. S. Desyatnikov, Flat band states: Disorder and nonlinearity, Phys. Rev. B 88, 224203 (2013).

[37] J. D. Bodyfelt, D. Leykam, C. Danieli, X. Yu, and S. Flach, Flatbands Under Correlated Perturbations, Phys. Rev. Lett. 113, 236403 (2014).

[38] C. Danieli, J. D. Bodyfelt, and S. Flach, Flat-band engineering of mobility edges, Phys. Rev. B 91, 235134 (2015).

[39] D. Leykam, J. D. Bodyfelt, A. S. Desyatnikov, and S. Flach, Localization of weakly disordered flat band states, Eur. Phys. J. B 90, 1 (2017).

[40] A. R. Kolovsky, A. Ramachandran, and S. Flach, Topological flat Wannier-Stark bands, Phys. Rev. B 97, 045120 (2018).

[41] C. Danieli, A. Maluckov, and S. Flach, Compact discrete breathers on flat-band networks, Low Temp. Phys. 44, 678 (2018). 
[42] A. Ramachandran, C. Danieli, and S. Flach, Fano resonances in flat band networks, in Fano Resonances in Optics and Microwaves: Physics and Applications, edited by Eugene Kamenetskii, Almas Sadreev, and Andrey Miroshnichenko (Springer International Publishing, Cham, 2018), pp. 311-329.

[43] J. Vidal, R. Mosseri, and B. Douçot, Aharonov-Bohm Cages in Two-Dimensional Structures, Phys. Rev. Lett. 81, 5888 (1998).

[44] J. Vidal, B. Douçot, R. Mosseri, and P. Butaud, Interaction Induced Delocalization for Two Particles in a Periodic Potential, Phys. Rev. Lett. 85, 3906 (2000).

[45] S. Tilleke, M. Daumann, and T. Dahm, Nearest neighbor particle-particle interaction in fermionic quasi one-dimensional flat band lattices, Z. Naturforsch., A 75, 393 (2020).

[46] C. Danieli, A. Andreanov, T. Mithun, and S. Flach, Caging of short-range interactions in all bands at lattices: Part I, arXiv:2004.11871; Caging of short-range interactions in all bands at lattices: Part II, arXiv:2004.11880.

[47] $\mathcal{H}_{\text {sp }}$ being fully or semidetangled and $\mathcal{H}_{\text {int }}$ being nondetangled.

[48] M. Serbyn, Z. Papić, and D. A. Abanin, Local Conservation Laws and the Structure of the Many-Body Localized States, Phys. Rev. Lett. 111, 127201 (2013).

[49] V. Ros, M. Müller, and A. Scardicchio, Integrals of motion in the many-body localized phase, Nucl. Phys. B 891, 420 (2015).

[50] J. M. Ziman, Principles of the Theory of Solids (Cambridge University Press, Cambridge, UK, 1972).

[51] S. Mukherjee and R. R. Thomson, Observation of localized flat-band modes in a quasi-one-dimensional photonic rhombic lattice, Opt. Lett. 40, 5443 (2015).

[52] S. Mukherjee and R. R. Thomson, Observation of robust flatband localization in driven photonic rhombic lattices, Opt. Lett. 42, 2243 (2017).

[53] S. Mukherjee, M. Di Liberto, P. Öhberg, R. R. Thomson, and N. Goldman, Experimental Observation of Aharonov-Bohm Cages in Photonic Lattices, Phys. Rev. Lett. 121, 075502 (2018).

[54] S. Xia, C. Danieli, W. Yan, D. Li, S. Xia, J. Ma, H. Lu, D. Song, L. Tang, S. Flach, and Z. Chen, Observation of quincunX-shaped and dipole-like flatband states in photonic rhombic lattices without band-touching, APL Photonics 5, 016107 (2020).

[55] M. Kremer, I. Petrides, E. Meyer, M. Heinrich, O. Zilberberg, and A. Szameit, A square-root topological insulator with non-quantized indices realized with photonic Aharonov-Bohm cages, Nat. Commun. 11, 907 (2020).

[56] We point out that unlike $1 \mathrm{D}$, the presence of several spatial directions may impose a constraint on the number of bands required to achieve MBFBL. For instance, the assumption of hopping between nearest-neighbor unit cells in $\mathcal{H}_{\mathrm{sp}}$ implies that 2D MBFBL networks have to have three or more bands.

[57] M. Röntgen, C. V. Morfonios, I. Brouzos, F. K. Diakonos, and P. Schmelcher, Quantum Network Transfer and Storage with Compact Localized States Induced by Local Symmetries, Phys. Rev. Lett. 123, 080504 (2019).

[58] Y. Kuno, T. Orito, and I. Ichinose, Flat-band many-body localization and ergodicity breaking in the Creutz ladder, New J. Phys. 22, 013032 (2020).

[59] J. Jünemann, A. Piga, S.-J. Ran, M. Lewenstein, M. Rizzi, and A. Bermudez, Exploring Interacting Topological Insulators with Ultracold Atoms: The Synthetic Creutz-Hubbard Model, Phys. Rev. X 7, 031057 (2017).

[60] R. Mondaini, G. G. Batrouni, and B. Grémaud, Pairing and superconductivity in the flat band: Creutz lattice, Phys. Rev. B 98, 155142 (2018).

[61] M. Tovmasyan, S. Peotta, L. Liang, P. Törmä, and S. D. Huber, Preformed pairs in flat Bloch bands, Phys. Rev. B 98, 134513 (2018).

[62] S. Barbarino, D. Rossini, M. Rizzi, R. Fazio, G. E. Santoro, and M. Dalmonte, Topological Devil's staircase in atomic two-leg ladders, New J. Phys. 21, 043048 (2019).

[63] N. Roy, A. Ramachandran, and A. Sharma, Compact, flat-band based, Anderson and many-body localization in a diamond chain, arXiv:1912.09951.

[64] T. Orito, Y. Kuno, and I. Ichinose, Exact projector Hamiltonian, local integrals of motion, and many-body localization with symmetry-protected topological order, Phys. Rev. B 101, 224308 (2020). 